\title{
Polygenic analysis of inflammatory disease variants and effects on microglia in the aging brain
}

Daniel Felsky ${ }^{1,2,3,4^{*}}$ (D), Ellis Patrick ${ }^{5}$, Julie A. Schneider ${ }^{6,7}$, Sara Mostafavi ${ }^{8}$, Chris Gaiteri ${ }^{6,7}$, Nikolaos Patsopoulos ${ }^{2,3}$, David A. Bennett ${ }^{6,7}$ and Philip L. De Jager ${ }^{1,2,3,4}$

\begin{abstract}
Background: The role of the innate immune system in Alzheimer's disease (AD) and neurodegenerative disease susceptibility has recently been highlighted in genetic studies. However, we do not know whether risk for inflammatory disease predisposes unaffected individuals to late-life cognitive deficits or AD-related neuropathology. We investigated whether genetic risk scores for seven immune diseases and central nervous system traits were related to cognitive decline $\left(n_{\max }=1601\right)$, classical AD neuropathology ( $\left.n_{\max }=985\right)$, or microglial density $\left(n_{\max }=184\right)$.

Methods: Longitudinal cognitive decline, postmortem amyloid and tau neuropathology, microglial density, and gene module expression from bulk brain tissue were all measured in participants from two large cohorts (the Rush Religious Orders Study and Memory and Aging Project; ROS/MAP) of elderly subjects (mean age at entry $78+/-8.7$ years). We analyzed data primarily using robust regression methods. Neuropathologists were blind to clinical data.

Results: The AD genetic risk scores, including and excluding APOE effects, were strongly associated with cognitive decline in all domains (min $P_{\text {uncor }}=3.2 \times 10^{-29}$ ). Multiple sclerosis $(M S)$, Parkinson's disease, and schizophrenia risk did not influence cognitive decline in older age, but the rheumatoid arthritis (RA) risk score alone was significantly associated with microglial density after correction $\left(t_{146}=-3.88, P_{\text {uncor }}=1.6 \times 10^{-4}\right)$. Post-hoc tests found significant effects of the RA genetic risk score in multiple regions and stages of microglial activation (min $P_{\text {uncor }}=1.5 \times 10^{-6}$ ). However, these associations were driven by only one or two variants, rather than cumulative polygenicity. Further, individual MS $\left(P_{\text {one-sided }}<8.4 \times 10^{-4}\right)$ and RA $\left(P_{\text {one-sided }}=3 \times 10^{-4}\right)$ variants associated with higher microglial density were also associated with increased expression of brain immune gene modules.

Conclusions: Our results demonstrate that global risk of inflammatory disease does not strongly influence agingrelated cognitive decline but that susceptibility variants that influence peripheral immune function also alter microglial density and immune gene expression in the aging brain, opening a new perspective on the control of microglial and immune responses within the central nervous system. Further study on the molecular mechanisms of peripheral immune disease risk influencing glial cell activation will be required to identify key regulators of these pathways.
\end{abstract}

Keywords: Genomics, Alzheimer's disease, Microglia, Inflammation, Polygenic score, Innate immunity, Postmortem, Neuropathology, RNA sequencing

\footnotetext{
* Correspondence: df2652@columbia.edu

${ }^{1}$ Center for Translational and Computational Neuroimmunology, Department of Neurology, Columbia University Medical Center, 630 West 168th Street, PH $19-302$, New York, NY 10032, USA

2Department of Neurology, Brigham and Woman's Hospital, 75 Francis Street, Boston, MA 02115, USA

Full list of author information is available at the end of the article
}

(c) The Author(s). 2018 Open Access This article is distributed under the terms of the Creative Commons Attribution 4.0 International License (http://creativecommons.org/licenses/by/4.0/), which permits unrestricted use, distribution, and reproduction in any medium, provided you give appropriate credit to the original author(s) and the source, provide a link to the Creative Commons license, and indicate if changes were made. The Creative Commons Public Domain Dedication waiver (http://creativecommons.org/publicdomain/zero/1.0/) applies to the data made available in this article, unless otherwise stated. 


\section{Background}

Genetic studies have highlighted the shared architecture of susceptibility among inflammatory diseases [1]; however, in unaffected individuals, the significance of this genetic predisposition to immune dysfunction for aging-related processes is unknown. Recent genetic studies of neurodegenerative diseases have also implicated the immune system - particularly the innate component - in susceptibility to aging-related conditions [2], with Alzheimer's disease (AD) susceptibility being most clearly enriched for variants that influence the expression and splicing of genes expressed in myeloid cells $[3,4]$. In the brain, microglia, the resident myeloid cells, have a prominent role, but infiltrating macrophages, $\mathrm{B}$ cells, and $\mathrm{T}$ cells also contribute to brain pathology in older age. Today, it is not clear whether a predisposition to pathologic inflammatory responses influences the function of organ systems in individuals that are not affected by an autoimmune disease. Here, we evaluate whether a propensity for excessive inflammation that has not manifested itself clinically in one's life course can influence the likelihood of (1) late-life cognitive deficits, (2) the accumulation of common neuropathology, including amyloid- $\beta$ $(\mathrm{A} \beta)$ and hyperphosphorylated tau, or (3) microglial recruitment and activation.

The genetic architecture of aging-related traits is not yet well understood; although their prevalence in the population and the heterogeneity observed among older individuals suggest that the allelic spectrum influencing susceptibility includes many common variants of modest effect [5-7]. To assess this hypothesis, we used a common strategy in which the effects of validated susceptibility variants are aggregated into a single additive genetic risk score (GRS). While useful as a screening tool, a GRS obscures the intrinsic granularity of the genomic risk landscape; therefore, they need to be complemented with targeted investigations of individual variants, where possible. We evaluated two representative inflammatory diseases for which the genetic architecture of susceptibility has been well described: MS, which targets the central nervous system, and rheumatoid arthritis (RA), for which a recent study of medical claims data from over 8.5 million adults reported an increased relative risk of AD among patients with RA. Further, anti-TNF $\alpha$ therapy for RA may lower this risk [8]. In MS, a neuropathological study reported no significant difference in the presence of AD pathologies compared to controls [9]; however, the prevalence of late-life pathologies and cognitive deficits in genetically-defined populations remains to be characterized.

We also evaluated GRS for four other traits that could influence brain aging and have been characterized genetically: Parkinson's disease (PD), telomere length, coronary artery disease (CAD), as well as schizophrenia, which may also have an inflammatory component [10]. Specifically, we deployed our GRS in two deeply characterized cohorts of aging individuals, deconstructed polygenic associations to resolve whether an association is driven by selected variants or a broad distribution of variants, and accessed cortical RNA sequence data to further develop our mechanistic understanding of observed genetic effects.

\section{Methods \\ Subjects}

Participants in this study were from the Religious Orders Study (ROS) [11] and the Rush Memory and Aging Project (MAP) [12], two cohort studies of elderly populations from the Chicago area conducted by investigators at the Rush Alzheimer's Disease Center (Rush University Medical Center, Chicago, IL, USA). All subjects were older and recruited free of dementia (mean age at entry $78+/-8.7$ years), agreed to annual clinical and neurocognitive evaluation, and signed an Anatomical Gift Act allowing for brain autopsy at time of death.

\section{Genetics and imputation}

In total, 1878 subjects were genotyped using the Affymetrix 6.0 Genechip. DNA was extracted from whole blood, lymphocytes, or frozen brain tissue and genotype data underwent standard quality control procedures using PLINK (v1.08), as previously described [13]. Briefly, subjects and variants were filtered based on genotype success rate $>0.95$, Hardy-Weinberg Equilibrium $P>0.001$, and mishap test $P>1 \times 10^{-9}$. After quality control of the initial genotype dataset, 1709 individuals and 750,173 autosomal variants remained. Whole genome imputation was performed using BEAGLE (v3.3.2) [14] and the 1000 Genomes reference panel (phase I haplotypes). To analyze the major histocompatibility complex (MHC) region in detail, a specialized imputation pipeline, SNP2HLA [15], was used. This was necessary given the major contribution of variation in this region to the pathogenesis of immune-related traits analyzed in this study. See Additional file 1: Supplementary Methods for details.

\section{Postmortem amyloid- $\beta$ and tau neuropathology}

Postmortem neuropathology data were available for up to 985 subjects at time of study. All brains were examined by a board-certified neuropathologist blinded to age and clinical data. $A \beta$ and abnormal tau deposition were measured using immunohistochemistry and automated image processing for total amyloid and paired helical filament tau, and a modified Bielschowsky silver staining technique for neuritic and diffuse plaques, and neurofibrillary tangles, as published previously [16]. 


\section{Postmortem microglial count density}

A subset of up to 183 brain samples with genomic data were evaluated for the presence of microglia at three stages of activation, based on morphology: stage 1 (thin ramified processes), stage 2 (plump cytoplasm and thicker processes), and stage 3 (appearance of macrophages). For each of four regions (midfrontal cortex, inferior temporal cortex, ventral medial caudate, and posterior putamen), four microglial density scores (total count of microglia/area surveyed) were calculated: stage 1 only, stage $1+2+3$, stage $2+3$, and stage 3 only [3] (see Additional file 1: Supplementary Methods).

\section{Cognitive decline}

A total of 1601 subjects with genomic data also had longitudinal cognitive performance data available at the time of study. ROS and MAP subjects were both administered 17 cognitive tests annually spanning five cognitive domains: episodic memory, semantic memory, working memory, perceptual speed, and visuospatial ability. Measures of cognitive performance for each domain were calculated by averaging z-scores across tests $[11,12,17]$, and rates of cognitive decline were calculated per subject using general linear mixed models of cognitive scores over time, co-varying for age at baseline, years of education, and sex, as described [6].

\section{Gene expression}

\section{RNA sequencing and post-processing}

RNA was extracted from DLPFC and sequenced on the Illumina HiSeq (50 million paired-end reads of $101 \mathrm{bp}$ each), as described [13]. Expression FPKM values were quantile-normalized, correcting for batch effect with Combat [18]. Paired-end reads were mapped to genes using the Ensemble human genome transcriptomic database (http://www.ensembl.org). Expression QTL (eQTL) analyses were performed in the ROS/MAP sample to ascertain potential mechanisms of pathology-associated gene variants with respect to gene expression [19]. The GTEx portal [20, 21] was used to corroborate eQTL effects.

\section{Clustering and module enrichment analyses}

Gene modules of co-expressed genes were derived using the SpeakEasy consensus clustering algorithm [22]. In ROS/MAP, SpeakEasy identified 47 mutually exclusive modules with 20-556 gene members (median=331), several of which have been shown to correlate strongly with pathology, cognition, and cell-type specific markers of gene expression in multiple datasets [23]. Of these modules, five (modules \#5, \#113, \#114, \#115, and \#116) show substantial enrichment for immune- and microglia-related functions and processes [23]. Immune gene modules were defined based on hypergeometric enrichment for microglia-specific genes (enrichment $p<$ 0.0011). These microglia-specific genes were defined based on Olah et al. (2018) [24] as at least four-fold upregulated in human bulk microglia. Module 113 (includes AD genes CLU, SPPL2A, SQSTM1, MPZL1, and ETS1) has an overlap of 24 microglial genes/313 total module genes $(P=0.0024)$; module 114 (no major $\mathrm{AD}$ genes) has an overlap of $24 / 276\left(P=4.5 \times 10^{-4}\right)$; module 115 (no major AD genes) has an overlap of 33/232 ( $P=$ $\left.4.1 \times 10^{-10}\right)$; module $116($ TREM2, INPP5D) has an overlap of $144 / 224\left(P=5.6 \times 10^{-148}\right)$; and module 5 (BIN1, PVRL2) has an overlap of 58/431 $\left(P=8.5 \times 10^{-16}\right)$. See Patrick et al. (2018) for details [25]. As such, expression levels of these five modules were used to benchmark the transcriptional effects of variants in functionally cohesive immune pathways.

\section{Statistical analysis \\ GRS calculation}

GRS were calculated using PLINK (v1.90b) [26] and all other analyses were performed using $\mathrm{R}$ (v3.3.3) [27]. We tested eight different GRS in this study: two inflammatory disease scores (MS and RA) and comparator scores that could influence aging-related cognitive decline, including those for $\mathrm{AD}$ (including $(+)$ and excluding $(-) A P O E$ ), PD (which frequently includes a dementing illness), CAD (the second most common cause of dementia in older individuals), schizophrenia (previously known as dementia praecox), and telomere length (a marker of biological aging). For each score, lists of genome-wide significant variants were extracted from state-of-the-art genome-wide association studies (listed in Table 1). Individual publications were chosen rather than an aggregate database to limit error due to between-study heterogeneity in outcome definitions, sample characteristics, and statistical methodology. The PLINK --score command was then used to generate average per-allele scores, weighted by each variant's published effect. Briefly, for each published trait, the number of effect alleles (i.e. those associated with an increase in trait outcome or disease liability) for each genome-wide significant variant was multiplied by its effect size (natural logarithm of the odds ratio or standardized beta coefficient), and these quantities were summed across variants within each subject to generate individual polygenic scores. Default parameters were used for this calculation, and missing genotypes contributed an amount to each score equal to the effect allele frequency in our sample, minimizing potential bias. Nonetheless, imputed genotype quality was high and each GRS calculation was manually inspected to ensure negligible and non-systematic missingness. 
Table 1 Summary of Studies Used to Derive Polygenic Scores

\begin{tabular}{lllll}
\hline Trait/Disease & Publication & $\begin{array}{l}\text { Total study size } \\
(\text { cases/controls) }\end{array}$ & \# of SNPs in score & $\begin{array}{l}\text { SNPs with ROS/MAP } \\
\text { MAF }>0.1\end{array}$ \\
\hline AD & Lambert et al., 2013 (Nat. Genet.) & $25,580 / 48,466$ & 22 & 18 \\
CAD & Nikpay et al., 2015 (Nat. Genet.) & $60,801 / 123,504$ & 63 & 54 \\
MS & Patsopoulos et al.,2017 (Biorxiv) & $47,351 / 68,284$ & 232 & 196 \\
PD & Nalls et al., 2014 (Nat. Genet.) & $13,708 / 95,282$ & 32 & 27 \\
RA & Okada et al., 2014 (Nature) & $29,880 / 73,758$ & 76 & 67 \\
Schizophrenia & Psychiatric Genomics Consortium, & $36,989 / 113,075$ & 106 & 100 \\
Telomere length & 2014 (Nature) & & 8 \\
\hline
\end{tabular}

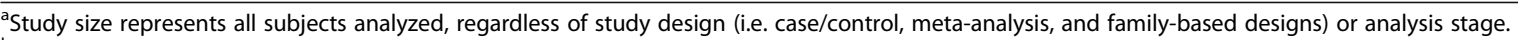

${ }^{b}$ The study by Codd et al., 2013 [43] was not a case/control design, as telomere length was evaluated as a continuous outcome. MAF = minor allele frequency. $\mathrm{MAP}=$ Rush Memory and Aging Project. ROS = Rush Religious Orders Study

\section{Associations of GRS with postmortem pathology and cognitive decline}

Associations of GRS with study outcomes were modeled using iterated re-weighted least squares, from the "MASS" $\mathrm{R}$ package [27]. The iterated re-weighted least squares regression technique employed here provides effect estimates robust to outliers by assigning weights to each observation and iteratively fitting Huber M-estimators [28]. All models of neuropathology co-varied for age at death, sex, postmortem interval, and the top three EIGENSTRAT principal components [29]. Tests were corrected using Benjamini \& Hochberg's false discovery rate (FDR) procedure [30]. Significant associations (two-sided $\left.P_{\text {FDR }}<0.05\right)$ were analyzed further to determine region and activation stage-specific effects on microglial density. All variants within each significantly associated GRS (with minor allele frequency $>0.1$ ) were then tested individually.

\section{Overlap between variants affecting microglial density and gene module expression}

To compare effects of risk variants on microglial density vs. effects of the same risk variants on gene module expression, the $-\log (P$-value $)$ for each variant's effect on both phenotypes were multiplied by their allelic directions of effect $(+1$ or -1$)$ and tested for association using Spearman rank correlations. Thus, whole sets of variants, grouped by the GRS to which they contribute, could be tested for synergistic effects on both microglial density and gene module expression. Correlation coefficients were calculated separately for each GRS and module combination, where positive $\rho$ values indicate a tendency for variants within a given GRS to influence both microglial density and gene module expression in concordant directions. The ranks of these coefficients for the five immune modules were then evaluated for significance by calculating the probability that the lowest of five randomly selected ranks would be equal to the lowest observed rank by chance alone (see Additional file 1: Supplementary Methods).

\section{Results}

The RA but not the MS GRS is associated with cognitive decline and postmortem neuropathology

Table 2 summarizes the demographic characteristics of the ROS/MAP participants that were included in our analyses. In comparing the GRS to one another, we found a modest correlation between the RA and MS scores $\left(\right.$ Spearman $\rho=-0.13, P_{\text {uncor }}=2.29 \times 10^{-8}$ ), as expected given the documented sharing of susceptibility loci between the two diseases (see Additional file 1: Figure S1).

After FDR correction, both the AD GRS including $\left(\mathrm{AD}^{+A P O E}\right)$ and excluding $\left(\mathrm{AD}^{-A P O E}\right)$ the $A P O E \varepsilon 4$ risk haplotype were significantly associated with faster decline in all cognitive domains proximal to death (min $\left.P_{\text {uncor }}<1 \times 10^{-16}\right)$, but no other GRS demonstrated significant associations (Fig. 1). The $\mathrm{AD}^{+A P O E}$ GRS was also strongly associated with both amyloid $\left(4.7 \times 10^{-21}>\right.$ $\left.P_{\text {uncor }}>1.8 \times 10^{-23}\right)$ and tau $\left(7.1 \times 10^{-20}>P_{\text {uncor }}>1.2 \times\right.$ $10^{-22}$ ) phenotypes; whereas the $\mathrm{AD}^{-A P O E}$ GRS was only associated with tau measures $\left(5.5 \times 10^{-4}>P_{\text {uncor }}>5.5 \times\right.$ $10^{-4}$ ) (Fig. 2). There were no associations of either AD GRS with microglial counts, confirming findings previously reported in these and other cohorts. [5, 31] Across all other scores, only the RA GRS was significantly associated with brain-wide microglial density after correction: an increase in liability for RA was associated with a decrease in microglial density $\left(\mathrm{t}_{146}=-3.88, P_{\text {uncor }}=\right.$ $1.6 \times 10^{-4}$ ) (Fig. 2). We then accessed our more detailed microglial data and repeated the analyses to test for the effects of each GRS on microglial count density across each brain region and stages of microglial activation. We found that the effect of the RA GRS is widely distributed, being present in multiple brain regions and stages of activation $\left(1.4 \times 10^{-3}>P_{\text {uncor }}>1.5 \times 10^{-6}\right)$. In addition, significant $\left(\mathrm{CAD} ; \mathrm{t}_{171}=3.44, P_{\text {uncor }}=7.3 \times 10^{-4}\right)$ and suggestive (MS and $\mathrm{AD}$ ) associations, particularly in relation to the activated stage 3 microglia, were noted in the inferior temporal gyrus (Fig. 3). 
Table 2 Sample Sizes and Characteristics for Each Analysis

\begin{tabular}{|c|c|c|c|c|c|}
\hline Phenotype & $\mathrm{N}$ & Mean & SD & Min & Max \\
\hline Neuritic Plaques & 985 & 0.86 & 0.85 & 0.00 & 5.04 \\
\hline Diffuse Plaques & & 0.73 & 0.77 & 0.00 & 4.61 \\
\hline Neurofibrillary Tangles & & 0.63 & 0.76 & 0.00 & 6.23 \\
\hline $\operatorname{Sex}(F / M)$ & & $641 / 344$ & - & - & - \\
\hline APOE $\varepsilon 4$ status $(-/+)$ & & $723 / 262$ & - & - & - \\
\hline Age at death & & 89.06 & 6.39 & 66.22 & 108.28 \\
\hline Dx at last visit (CN/MCl/AD/other) & & $319 / 237 / 343 / 86$ & - & - & - \\
\hline PMl & & 8.57 & 7.59 & 0.00 & 85.08 \\
\hline Total Amyloid & 952 & 4.21 & 4.20 & 0.00 & 19.93 \\
\hline $\operatorname{Sex}(F / M)$ & & $617 / 335$ & - & - & - \\
\hline APOE $\varepsilon 4$ status $(-/+)$ & & $697 / 255$ & - & - & - \\
\hline Age at death & & 88.95 & 6.38 & 66.22 & 108.28 \\
\hline Dx at last visit (CN/MCl/AD/other) & & $309 / 230 / 329 / 84$ & - & - & - \\
\hline PMl & & 8.52 & 7.57 & 0.00 & 85.08 \\
\hline Total PHF-Tau & 946 & 6.43 & 7.70 & 0.00 & 78.52 \\
\hline $\operatorname{Sex}(F / M)$ & & $615 / 331$ & - & - & - \\
\hline APOE $\varepsilon 4$ status $(-/+)$ & & $694 / 252$ & - & - & - \\
\hline Age at death & & 88.91 & 6.38 & 66.22 & 108.28 \\
\hline Dx at last visit (CN/MCl/AD/other) & & $312 / 228 / 326 / 80$ & - & - & - \\
\hline PMl & & 8.47 & 7.56 & 0.00 & 85.08 \\
\hline Microglial Density (all regions) & 154 & 191.02 & 54.88 & 48.30 & 348.64 \\
\hline $\operatorname{Sex}(F / M)$ & & $96 / 58$ & - & - & - \\
\hline APOE $\varepsilon 4$ status $(-/+)$ & & $117 / 37$ & - & - & - \\
\hline Age at death & & 89.50 & 5.15 & 74.83 & 101.19 \\
\hline Dx at last visit (CN/MCl/AD/other) & & $51 / 41 / 58 / 4$ & - & - & - \\
\hline PMl & & 7.36 & 5.97 & 2.50 & 54.50 \\
\hline Cognition & 1601 & -0.01 & 0.09 & -0.48 & 0.17 \\
\hline $\operatorname{Sex}(F / M)$ & & $1113 / 488$ & - & - & - \\
\hline APOE $\varepsilon 4$ status $(-/+)$ & $1594^{a}$ & $1186 / 408$ & - & - & - \\
\hline Age at baseline evaluation & 1601 & 86.50 & 6.81 & 60.15 & 108.15 \\
\hline Dx at last visit (CN/MCl/AD/other) & & $700 / 357 / 436 / 108$ & - & - & - \\
\hline
\end{tabular}

Note: All values of $\mathrm{N}$ are given for samples that have data for both the specified phenotype and genome-wide genotypes.

${ }^{a} A P O E \varepsilon 4$ status was obtained separately from genome-wide genotypes, so seven samples with cognitive data did not have APOE $\varepsilon 4$ status data available at time of study, $C N$ cognitively normal, Dx diagnosis, $F$ female, $M$ male, $M C I$ mild cognitive impairment, PHF-Tau paired helical filament tau, $P M I$ postmortem interval, SD standard deviation

\section{Individual variants drive GRS associations with microglial density}

To understand what drives the association of the RA and CAD GRS with microglial phenotypes, we analyzed individual variants contributing to each GRS. For CAD, rs4977574 (chr. 9) alone drove the GRS association, and, for RA, two variants, rs9268839 (chr. 6) and rs10175798 (chr. 2), were responsible (Fig. 4). After removal of these two top RA GRS variants and re-calculation of the RA score, we found no association with total microglial density $(P=0.74)$ nor with any of the region- or stage-specific measures $(0.23>P<0.88)$. The more strongly associated RA variant, rs9268839, is found in the Human Leukocyte Antigen (HLA) class II region and is also the strongest RA susceptibility variant in Europeans $\quad$ O.R. $=2.47, \quad$ C.I. $95 \%=[2.39,2.55], \quad P_{\text {meta }}=$ $\left.1.5 \times 10^{-300}\right)$ [32]. Therefore, upweighting of this variant in the calculation of the RA GRS biased its effect on microglial density (Additional file 1: Figure S3). In the CAD GRS, a similar pattern was observed: rs4977574 drives the association and is the strongest hit in the CAD genome-wide association study (O.R. $=1.21$, C.I.95\% $=[1.19,1.24], P_{\text {meta }}=2.29 \times 10^{-98}$ ) [33] (Additional file 1: Figure S4). 


\begin{tabular}{|c|c|c|c|c|c|c|c|c|c|c|}
\hline \multirow{7}{*}{ 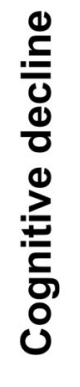 } & Global cognition & $2 e-05^{*}$ & $3.2 e-29^{*}$ & 0.54 & 0.45 & 0.71 & 0.93 & 0.42 & 0.49 & \multirow{6}{*}{$\begin{array}{c}\text { T-stat } \\
0 \\
-3 \\
-6 \\
-9\end{array}$} \\
\hline & Episodic memory & $8.3 e-05^{\star}$ & $2 e-28^{*}$ & 0.54 & 0.49 & 0.53 & 0.56 & 0.26 & 0.41 & \\
\hline & \multirow{4}{*}{$\begin{array}{l}\text { Visuo-spatial ability } \\
\text { Processing speed } \\
\text { Semantic memory } \\
\text { Working memory }\end{array}$} & $0.0041^{*}$ & $2.1 \mathrm{e}-08^{*}$ & 0.56 & 0.76 & 0.7 & 0.92 & 0.15 & 0.33 & \\
\hline & & $2.4 \mathrm{e}-05^{*}$ & $2.4 e-14 *$ & 0.5 & 0.97 & 0.65 & 0.97 & 0.57 & 0.37 & \\
\hline & & $7.3 e-05^{\star}$ & $1.4 e-24 *$ & 0.84 & 0.66 & 0.93 & 0.9 & 0.3 & 0.51 & \\
\hline & & $0.0027^{*}$ & $3.9 e-16^{*}$ & 0.53 & 0.69 & 0.9 & 0.87 & 0.58 & 0.68 & \\
\hline & & $\begin{array}{c}\mathrm{AD} \\
-A P O E\end{array}$ & $\begin{array}{c}\mathrm{AD} \\
+A P O E\end{array}$ & CAD & MS & PD & $\mathrm{RA}$ & SCZ & TEL & \\
\hline \multicolumn{11}{|c|}{ Polygenic Score } \\
\hline \multicolumn{11}{|c|}{$\begin{array}{l}\text { Fig. } 1 \text { Analysis of GRS vs. cognitive decline slopes }(n=1601) \text {. Two-sided uncorrected } P \text {-values derived from robust regression are shown within } \\
\text { tiles. Models co-varied for age at initial assessment, sex, years of education, and three EIGENSTRAT principal components. The color scale indicates } \\
\text { magnitude and direction of the effect T-statistic. *significant after FDR correction }\left(P_{\text {FDR }}<0.05\right)\end{array}$} \\
\hline
\end{tabular}

In ROS/MAP cortical RNA and GTEx, the top variant influencing microglial density for the RA score (rs9268839, $P_{\text {uncor }}$ $=2.48 \times 10^{-5}$ ) influences HLA class II expression (see Additional file 1: Supplementary Methods). The top CAD SNP, rs4977574, has no cis-eQTL effects in these data but has been reported to influence VIL2 in blood $\left(P=6.1 \times 10^{-6}\right)$ [34].
Variants affecting microglial density also tend to affect expression of immune gene modules

Another approach to exploring the role of GRS in the aging brain involves assessing their effects on the cortical transcriptome of ROS/MAP participants, using the 47 modules of co-expressed genes previously defined in

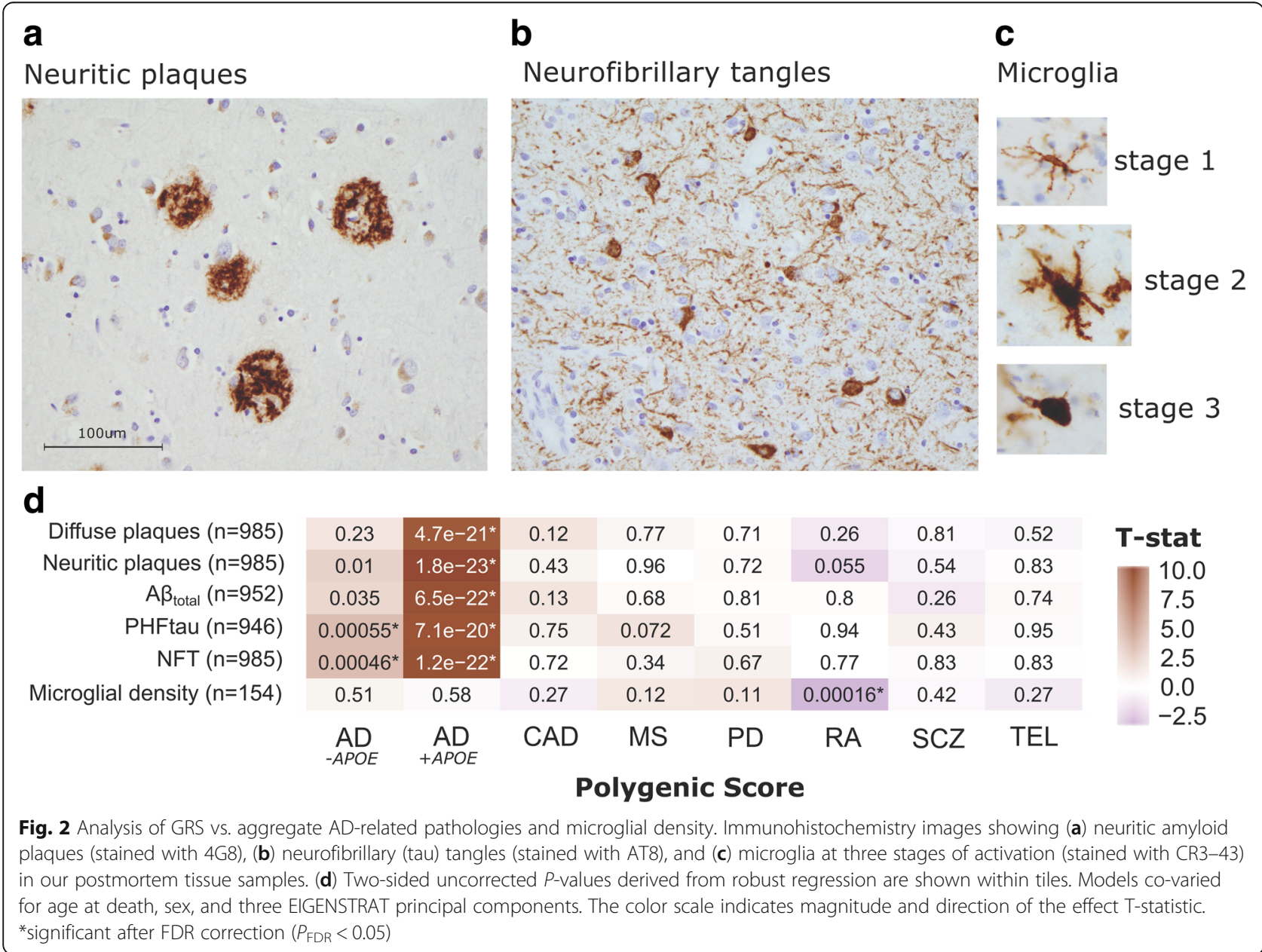




\begin{tabular}{|c|c|c|c|c|c|c|c|c|c|c|}
\hline & 3 & 0.97 & 0.95 & 0.37 & 0.026 & 0.7 & $1 e-05^{*}$ & 0.34 & 0.26 & \\
\hline ver & $2+3$ & 0.89 & 0.97 & 0.56 & 0.02 & 0.79 & $1.5 e-06^{*}$ & 0.63 & 0.2 & \\
\hline & $1+2+3$ & 0.52 & 0.91 & 0.41 & 0.061 & 0.72 & $0.00015^{*}$ & 0.57 & 0.6 & \\
\hline & 1 & 0.45 & 0.97 & 0.51 & 0.085 & 0.64 & $3 e-04^{*}$ & 0.65 & 0.64 & \\
\hline & 3 & 0.24 & 0.25 & 0.63 & 0.19 & 0.92 & 0.013 & 0.97 & 0.26 & \\
\hline & $2+3$ & 0.32 & 0.28 & 0.86 & 0.075 & 0.92 & $0.00069^{*}$ & 0.8 & 0.35 & \\
\hline & $1+2+3$ & 0.46 & 0.72 & 0.58 & 0.15 & 0.19 & $0.00062^{*}$ & 0.56 & 0.28 & -stat \\
\hline & 1 & 0.4 & 0.7 & 0.55 & 0.16 & 0.19 & $0.0014^{*}$ & 0.56 & 0.35 & 2 \\
\hline & 3 & 0.44 & 0.84 & 0.2 & 0.043 & 0.56 & $0.0013^{*}$ & 0.79 & 0.28 & 0 \\
\hline & $2+3$ & 0.52 & 0.55 & 0.66 & 0.057 & 0.2 & $0.00072^{*}$ & 1 & 0.33 & -2 \\
\hline & $1+2+3$ & 0.47 & 0.24 & 0.45 & 0.12 & 0.28 & $5.4 e-05^{*}$ & 0.46 & 0.45 & -4 \\
\hline & 1 & 0.43 & 0.2 & 0.34 & 0.16 & 0.24 & $7.7 e-05^{*}$ & 0.43 & 0.47 & \\
\hline & 3 & 0.02 & 0.013 & $0.00073^{*}$ & 0.0093 & 0.39 & 0.034 & 0.65 & 0.22 & \\
\hline inter & $2+3$ & 0.017 & 0.023 & 0.062 & 0.013 & 0.48 & 0.1 & 0.59 & 0.26 & \\
\hline$=11^{\circ}$ & $1+2+3$ & 0.56 & 0.99 & 0.73 & 0.59 & 0.046 & 0.0091 & 0.43 & 0.059 & \\
\hline & 1 & 0.69 & 0.79 & 0.64 & 0.76 & 0.045 & 0.012 & 0.49 & 0.07 & \\
\hline Region & Stage & $\underset{-A P O E}{A D}$ & $\begin{array}{c}\mathrm{AD} \\
+A P O E\end{array}$ & CAD & MS & PD & RA & SCZ & TEL & \\
\hline \multicolumn{11}{|c|}{ Polygenic Score } \\
\hline $\begin{array}{l}\text { Fig. } \\
\text { from } \\
\text { scale }\end{array}$ & $\begin{array}{l}\text { GRS v } \\
\text { ssion a }\end{array}$ & $\begin{array}{l}\text { oglial } \\
\text { wn wi }\end{array}$ & $\begin{array}{l}\text { acr } \\
\text { s. Mc }\end{array}$ & reg & $\begin{array}{l}\text { each } \\
\text { ge at } \\
\text { ifican }\end{array}$ & $\begin{array}{l}d \text { st } \\
\text { ex, a } \\
\text { DR c }\end{array}$ & $\begin{array}{l}\text { activati } \\
\text { e. ElGE } \\
\text { in ( } P_{\text {FDP }}\end{array}$ & $\begin{array}{l}\text { (O-side } \\
\text { T) prin } \\
\text { 5) }\end{array}$ & $\begin{array}{l}\text { recte } \\
\text { mpo }\end{array}$ & $\begin{array}{l}\text { derived } \\
\text { color }\end{array}$ \\
\hline
\end{tabular}

these individuals [23]. Additional file 1: Figure S5A shows the results of the direct association of each gene module's expression with each GRS. After correction, this analysis yielded no significant results overall; however, 17 correlations exceeded $P_{\text {uncor }}<$ 0.05 . Evaluating gene module expression directly against midfrontal microglia density (stages $1+2+3$ ), only module \#118 had a significant effect after correction $\left(\mathrm{t}_{94}=-3.60, P_{\text {uncor }}=5.1 \times 10^{-4}\right)$, whereby increased expression was associated with a decrease in microglial density (Additional file 1: Figure S5B). To evaluate the intersection of gene variant effects on both microglial density and gene expression, Spearman correlations of variant effects on both outcomes were evaluated within each GRS. Again, no results were significant after correction, largely due to a sparsity of individually significant associations of GRS SNPs with either microglial density or module expression. Nonetheless, our exploratory findings suggest a possible tendency for variants which affect microglial density to also influence immune module expression (See Additional file 1: Figure S7, Additional file 1: Supplementary Results).

\section{Discussion}

We show that polygenic risk burden for RA and CAD significantly impact microglial count density, in different regions and at different stages of activation, in postmortem brain of elderly individuals. However, these associations were driven by only one or two variants within each GRS, highlighting a key limitation in the use of polygenic risk models of complex traits. In joint analyses of GRS, microglial densities, and RNA sequencing from the frontal cortex, we found no significant direct associations between GRS and immune gene module expression. However, when evaluating pleiotropy among GRS variants, microglial density and immune gene expression, we noted significant associations of MS, CAD, and RA risk variants with brain-wide microglial density and expression of at least one immune module. In parallel, a high genetic MS burden was linked to a loss of modules that are enriched for neuronal or mitochondrial genes, suggesting that it may play a role in exacerbating the dysfunction or loss of neurons or their transcriptional programs.

Several GRS tested showed effects in the directions expected based on existing literature: both the $\mathrm{AD}^{+A P O E}$ 


\section{Ventral medial caudate (stages $2+3$ )}

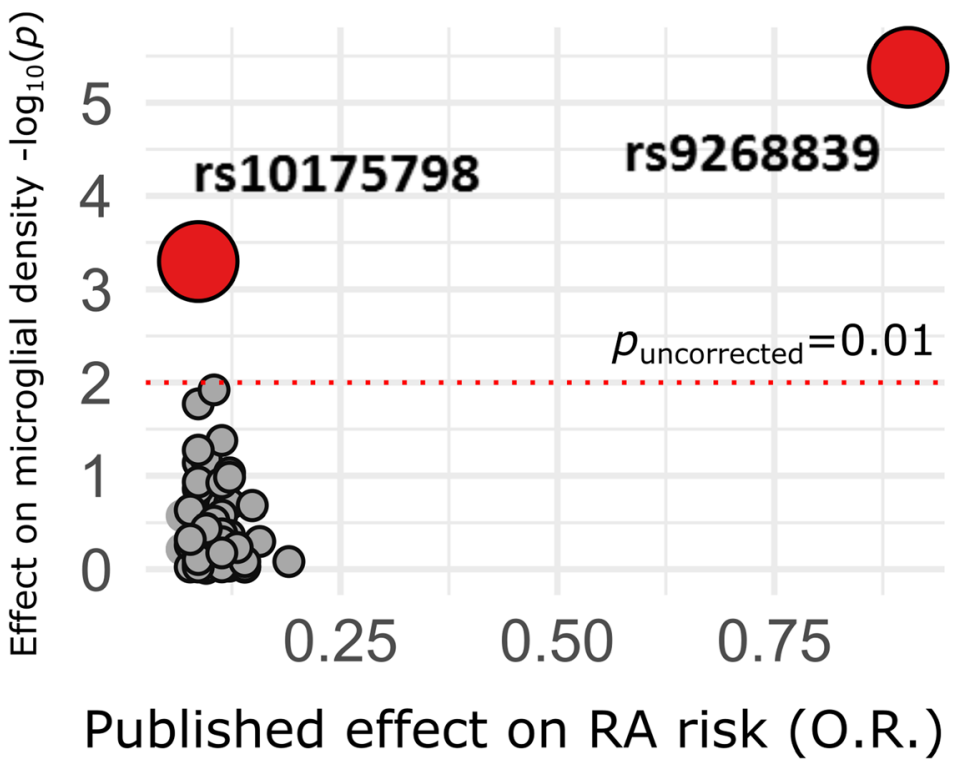

\section{Direction of Variant Effect \\ -ve}

Fig. 4 Analysis of individual variants in the RA GRS on microglial density in the ventral medial caudate. Published effect sizes on the x-axis have been transformed using a natural logarithm and oriented in the positive direction to align allelic effects (color denotes direction of effect on microglial density). $P$-values (uncorrected) are two-sided and derived from robust iterated re-weighted least squares regression models, co-varying for age at death, sex, and three EIGENSTRAT principal components

and $\mathrm{AD}^{-A P O E}$ GRS were significantly associated with cognitive decline at corrected thresholds, but, interestingly, the $\mathrm{AD}^{-A P O E}$ GRS was only significantly associated with tau-related neuropathology, complimenting existing evidence from in vivo PET imaging and CSF analyses showing an effect of $A P O E \& 4$ on amyloid- but not tau-related biomarkers in healthy elderly [35]. While our observed association of the CAD GRS with microglial density in the inferior temporal cortex was not expected, the variant driving this association, rs4977574, is in high linkage disequilibrium $\left(\mathrm{r}^{2}=0.89\right)$ with another variant, rs1333049, that has been associated at genome-wide significance with risk for ischemic stroke [36]. It is possible that the overlapping susceptibility at this locus for CAD and stroke drives cerebrovascular changes that lead to the recruitment and activation of microglia. Analyses of regional interactions between microglia density and other types of brain pathology, such as silent infarction, is beyond the scope of our current study and is a topic of future interest. For our observed association of the RA GRS with microglia across multiple activation stages, there appears to be little regional specificity other than that the association is much less pronounced is the inferior temporal gyrus. Interestingly, this region is the earliest affected in $\mathrm{AD}$ [37] and appears to behave differently from the other three brain regions in our analyses: it harbors additional, significant (CAD) and suggestive (MS and AD) associations in secondary analyses, particularly in relation to the activated, stage 3 microglia. Finally, we note that, while we elected to use an FDR-based correction in our original analysis plan, our main results also meet more conservative thresholds of significance, such as Bonferroni correction.

Many tests revealed a lack of GRS effects on our outcomes: for example, the schizophrenia GRS was not associated with any measure of cognitive decline or neuropathology. This seemingly contradicts previous evidence of increased HLA-DR ${ }^{+}$microglia in brains of schizophrenia patients compared to age-matched controls [38]. Our lack of association of schizophrenia GRS with microglial density in any area and at any stage of activation suggests that the mechanism behind aberrant microglial recruitment in the schizophrenia brain is less likely to be due to schizophrenia-specific genetic risk factors; rather, it may be driven by environmental factors or be a consequence of processes downstream of the onset of schizophrenia. In addition, for telomere length variants, we find a lack of association with AD pathology or cognitive decline, in contrast to a recent meta-analysis finding that $\mathrm{AD}$ patients have shorter telomeres than controls 
[39], as well as Mendelian randomization analyses suggesting causal links between telomere length and $\mathrm{AD}$ [40]. Also, our top signals for association across all GRS (including RA) localized to the MHC region, which influences many immune traits, and resolving the mechanism of this association to the HLA class II region within the MHC will be difficult given the extensive linkage disequilibrium that exists in this unique genomic region [41].

The main limitation of our GRS is that they were derived from lists of genome-wide significant loci only. We chose this approach due to (1) the lack of unrestricted availability of full summary statistics for all diseases tested, and (2) validity assumptions of individual variant analyses. If many variants well below genome-wide significance from each GWAS were included in our GRS calculations, then post-hoc associations of individual variants may not be relevant in the context of risk for the disease of interest. Moreover, our post-mortem measure of microglial activation is based on morphologic criteria and is subject to error associated with misclassification of individual cells to specific microglial stages of activation. However, by analyzing multiple binned groups of microglia by stage in our detailed analyses, the confounding of misclassification over the spectrum of groups has likely been mitigated. Also, the challenge of objectively classifying microglial activation states is not unique to our study; microglial staging is an active field of investigation [42].

\section{Conclusions}

Together, our findings demonstrate limited links between microglial activation and liability for archetypal inflammatory diseases of both the central nervous system (MS) and periphery (RA). Thus, the immune dysfunction involved in AD susceptibility seems to be largely distinct from those genes and pathways that are involved in susceptibility to inflammatory disease in young and middle-aged adults. Nonetheless, we have uncovered a handful of variants that have strong effects on both inflammatory disease risk and microglial density, which informs our understanding of human microglial biology in aging which remains poorly understood today.

\section{Additional file}

Additional file 1: Supplementary Methods, Results, and Figures. (DOCX 1972 kb)

\section{Abbreviations}

AD: Alzheimer's disease; A $\mathrm{\beta}$ : Amyloid beta; CAD: Coronary artery disease; CSF: cerebrospinal fluid; eQTL: Expression quantitative trait locus/loci; FDR: False discovery rate; FPKM: Fragments per kilobase per million reads; GRS: Genetic risk score; GWAS: Genome-wide association study; HLA: Human leukocyte antigen; MAP: Memory and Aging Project; MHC: Major histocompatibility complex; MS: Multiple sclerosis; PD: Parkinson's disease; PET: Positron emissions tomography; PMI: Postmortem interval; RA: Rheumatoid arthritis; ROS: Religious Orders Study

\section{Acknowledgements}

We thank the participants in the Religious Orders Study and the Rush Memory and Aging Project.

\section{Funding}

This study was supported by NIH grants P30AG10161, R01AG15819, R01AG17917, R01AG30146, R01NS084965, the Illinois Department of Public Health, and the Translational Genomics Research Institute. D.F. is supported by a Canadian Institutes of Health Research Postdoctoral Fellowship. No funding source played a role in the design or analysis of the study, nor was involved in the writing of the manuscript.

\section{Availability of data and materials}

The datasets generated and/or analysed during the current study are available in the Rush Alzheimer's Disease Center Resource Sharing Hub (https://www.radc.rush.edu/).

\section{Authors' contributions}

DF was responsible for study design, data management and pre-processing, all statistical analyses, and writing of the manuscript. EP contributed to the study design, post-processing of RNA sequencing data, and manuscript editing. JAS was responsible for overseeing neuropathological data acquisition, ensuring quality control of the data, and editing the final manuscript. SM and CG were responsible for calculating gene module meta-feature values and editing final manuscript. NP contributed HLA region imputed data as well as important information regarding MS risk variants. DAB contributed to the study design and manuscript editing, as well as facilitating collaboration between study sites. PLDJ contributed to the study design, evaluation of results, and writing of the manuscript. All authors read and approved the final manuscript.

\section{Ethics approval and consent to participate}

The Institutional Review Board of Rush University Medical Center approved study protocols and all subjects provided informed, written consent, and signed an Anatomical Gift Act.

\section{Consent for publication}

Not applicable.

\section{Competing interests}

The authors declare that they have no competing interests.

\section{Publisher's Note}

Springer Nature remains neutral with regard to jurisdictional claims in published maps and institutional affiliations.

\section{Author details}

${ }^{1}$ Center for Translational and Computational Neuroimmunology, Department of Neurology, Columbia University Medical Center, 630 West 168th Street, PH 19 - 302, New York, NY 10032, USA. ²Department of Neurology, Brigham and Woman's Hospital, 75 Francis Street, Boston, MA 02115, USA. ${ }^{3}$ Department of Neurology, Harvard Medical School, 25 Shattuck Street, Boston, MA 02115, USA. ${ }^{4}$ Program in Population and Medical Genetics, Broad Institute of MIT and Harvard, 415 Main St, Cambridge, MA 02142, USA. ${ }^{5}$ Department of Statistics, University of Sydney, Camperdown, NSW 2006, Australia.

${ }^{6}$ Department of Neurology, Rush University Medical Center, 1653 West Congress Parkway, Chicago, IL 60612, USA. ${ }^{7}$ Rush Alzheimer's Disease Center, Rush University Medical Center, 600 South Paulina Street, Chicago, IL 60612, USA. ${ }^{8}$ Department of Statistics, University of British Columbia, 2329 West Mall, Vancouver, BC V6T 1Z4, Canada.

Received: 10 November 2017 Accepted: 13 July 2018 Published online: 24 July 2018

\section{References}

1. Parkes M, Cortes A, van Heel DA, Brown MA. Genetic insights into common pathways and complex relationships among immune-mediated diseases. Nat Rev Genet. 2013;14:661-73.

2. Raj T, Rothamel K, Mostafavi S, Ye C, Lee MN, Replogle JM, et al. Polarization of the effects of autoimmune and neurodegenerative risk alleles in leukocytes. Science. 2014;344:519-23. 
3. Bradshaw EM, Chibnik LB, Keenan BT, Ottoboni L, Raj T, Tang A, et al. CD33 Alzheimer's disease locus: altered monocyte function and amyloid biology. Nat Neurosci. 2013;16:848-50.

4. Huang K, Marcora E, Pimenova A, Narzo AD, Kapoor M, Jin SC, et al. A common haplotype lowers PU.1 expression in myeloid cells and delays onset of Alzheimer's disease. Nat Neurosci. 2017;8;1052-61.

5. Beecham GW, Hamilton K, Naj AC, Martin ER, Huentelman M, Myers AJ, et al. Genome-wide association meta-analysis of Neuropathologic features of Alzheimer's disease and related dementias. PLoS Genet. 2014;10:e1004606.

6. De Jager PL, Shulman JM, Chibnik LB, Keenan BT, Raj T, Wilson RS, et al. A genome-wide scan for common variants affecting the rate of age-related cognitive decline. Neurobiol Aging. 2012;33:1017.e1-15.

7. Chibnik LB, White CC, Mukherjee S, Raj T, Yu L, Larson EB, et al. Susceptibility to neurofibrillary tangles: role of the PTPRD locus and limited pleiotropy with other neuropathologies. Mol Psychiatry. 2017;23(6):1521-9.

8. Chou RC, Kane M, Ghimire S, Gautam S, Gui J. Treatment for rheumatoid arthritis and risk of Alzheimer's disease: a nested case-control analysis. CNS Drugs. 2016;30:1111-20.

9. Dal Bianco A, Bradl M, Frischer J, Kutzelnigg A, Jellinger $K$, Lassmann $H$. Multiple sclerosis and Alzheimer's disease. Ann Neurol. 2008;63:174-83.

10. Schizophrenia Working Group of the Psychiatric Genomics Consortium. Biological insights from 108 schizophrenia-associated genetic loci. Nature. 2014;511:421-7.

11. Bennett DA, Schneider JA, Arvanitakis Z, Wilson RS. Overview and findings from the religious orders study. Curr Alzheimer Res. 2012;9:628-45.

12. Bennett DA, Schneider JA, Buchman AS, Barnes LL, Boyle PA, Wilson RS. Overview and findings from the rush memory and aging project. Curr Alzheimer Res. 2012;9:646-63.

13. Bennett DA, Yu L, De Jager PL. Building a pipeline to discover and validate novel therapeutic targets and lead compounds for Alzheimer's disease. Biochem Pharmacol. 2014;88:617-30.

14. Browning BL, Browning SR. A unified approach to genotype imputation and haplotype-phase inference for large data sets of trios and unrelated individuals. Am J Hum Genet. 2009;84:210-23.

15. Jia X, Han B, Onengut-Gumuscu S, Chen W-M, Concannon PJ, Rich SS, et al. Imputing amino acid polymorphisms in human leukocyte antigens. PLoS One. 2013;8:e64683.

16. Bennett DA, Wilson RS, Boyle PA, Buchman AS, Schneider JA. Relation of neuropathology to cognition in persons without cognitive impairment. Ann Neurol. 2012;72:599-609.

17. Bennett DA, Wilson RS, Schneider JA, Evans DA, Beckett LA, Aggarwal NT, et al. Natural history of mild cognitive impairment in older persons. Neurology. 2002;59:198-205.

18. Johnson WE, Li C, Rabinovic A. Adjusting batch effects in microarray expression data using empirical Bayes methods. Biostat Oxf Engl. 2007:8:118-27.

19. Ng B, White CC, Klein HU, Sieberts SK, MCCabe C, Patrick E, et al. Brain XQTL Map: Integrating The Genetic Architecture Of The Human Brain Transcriptome And Epigenome. bioRxiv. 2017;142927

20. Lonsdale J, Thomas J, Salvatore M, Phillips R, Lo E, Shad S, et al. The genotype-tissue expression (GTEx) project. Nat Genet. 2013;45:580-5.

21. The GTEx Consortium. The genotype-tissue expression (GTEx) pilot analysis: multitissue gene regulation in humans. Science. 2015;348:648-60.

22. Gaiteri C, Chen M, Szymanski B, Kuzmin K, Xie J, Lee C, et al. Identifying robust communities and multi-community nodes by combining top-down and bottom-up approaches to clustering. ArXiv150104709 Phys Q-Bio [Internet]. 2015 [cited 2015 Sep 13]; Available from: http://arxiv.org/abs/ 1501.04709

23. Mostafavi S, Gaiteri C, Sullivan S, White C, Takasi S, Xu J, et al. A molecular network of the aging brain implicates INPPL1 and PLXNB1 in Alzheimer's disease. bioRxiv. 2017;205807

24. Olah M, Patrick E, Villani A-C, Xu J, White CC, Ryan KJ, et al. A transcriptomic atlas of aged human microglia. Nat Commun. 2018;9:539.

25. Patrick E, Olah M, Taga M, Klein H-U, Xu J, White CC, et al. A cortical immune network map identifies a subset of human microglia involved in Tau pathology. bioRxiv. 2017;234351

26. Purcell S, Neale B, Todd-Brown K, Thomas L, Ferreira MAR, Bender D, et al. PLINK: a tool set for whole-genome association and population-based linkage analyses. Am J Hum Genet. 2007;81:559-75.

27. R Core Team. R: A language and environment for statistical computing [Internet]. Vienna: Vienna, Austria: R Foundation For Statistical Computing; 2014. Available from: http://www.r-project.org/
28. Huber PJ. Robust statistics [Internet]. 1981 [cited 2017 May 2]. Available from: https://zbmath.org/?q=an:0536.62025

29. Price AL, Patterson NJ, Plenge RM, Weinblatt ME, Shadick NA, Reich D. Principal components analysis corrects for stratification in genome-wide association studies. Nat Genet. 2006;38:904-9.

30. Benjamini $Y$, Hochberg Y. Controlling the false discovery rate: a practical and powerful approach to multiple testing. J R Stat Soc Ser B Methodol. 1995;57:289-300.

31. Mormino EC, Sperling RA, Holmes AJ, Buckner RL, De Jager PL, Smoller JW, et al. Polygenic risk of Alzheimer disease is associated with early- and latelife processes. Neurology. 2016;87:481-8.

32. Okada Y, Wu D, Trynka G, Raj T, Terao C, Ikari K, et al. Genetics of rheumatoid arthritis contributes to biology and drug discovery. Nature. 2014;506:376-81.

33. Nikpay M, Goel A, Won H-H, Hall LM, Willenborg C, Kanoni S, et al. A comprehensive 1000 genomes-based genome-wide association metaanalysis of coronary artery disease. Nat Genet. 2015;47:1121-30.

34. Fehrmann RSN, Jansen RC, Veldink JH, Westra H-J, Arends D, Bonder MJ, et al. Trans-eQTLs reveal that independent genetic variants associated with a complex phenotype converge on intermediate genes, with a major role for the HLA. PLoS Genet. 2011;7:e1002197.

35. Morris JC, Roe CM, Xiong C, Fagan AM, Goate AM, Holtzman DM, et al. APOE predicts amyloid-beta but not tau Alzheimer pathology in cognitively normal aging. Ann Neurol. 2010;67:122-31.

36. Dichgans M, Malik R, König IR, Rosand J, Clarke R, Gretarsdottir S, et al. Shared genetic susceptibility to ischemic stroke and coronary artery disease: a genome-wide analysis of common variants. Stroke. 2014;45:24-36.

37. Braak H, Braak E. Neuropathological stageing of Alzheimer-related changes. Acta Neuropathol (Berl). 1991;82:239-59.

38. Radewicz K, Garey L, Gentleman SM, Reynolds R. Increase in HLA-DR Immunoreactive microglia in frontal and temporal cortex of chronic schizophrenics. J Neuropathol Exp Neurol. 2000;59:137-50.

39. Forero DA, González-Giraldo Y, López-Quintero C, Castro-Vega LJ, Barreto GE, Perry G. Meta-analysis of telomere length in Alzheimer's disease. J Gerontol A Biol Sci Med Sci. 2016;71:1069-73.

40. Zhan Y, Song C, Karlsson R, Tillander A, Reynolds CA, Pedersen NL, et al. Telomere length shortening and Alzheimer disease-a Mendelian randomization study. JAMA Neurol. 2015;72:1202-3.

41. Shiina T, Hosomichi K, Inoko H, Kulski JK. The HLA genomic loci map: expression, interaction, diversity and disease. J Hum Genet. 2009;54:15-39.

42. Boche D, Perry VH, Nicoll J. a. R. Review: activation patterns of microglia and their identification in the human brain. Neuropathol Appl Neurobiol. 2013; 39:3-18.

43. Codd V, Nelson CP, Albrecht E, Mangino M, Deelen J, Buxton JL, et al. Identification of seven loci affecting mean telomere length and their association with disease. Nat Genet. 2013:45:422-427e2.

Ready to submit your research? Choose BMC and benefit from:

- fast, convenient online submission

- thorough peer review by experienced researchers in your field

- rapid publication on acceptance

- support for research data, including large and complex data types

- gold Open Access which fosters wider collaboration and increased citations

- maximum visibility for your research: over $100 \mathrm{M}$ website views per year

At $\mathrm{BMC}$, research is always in progress.

Learn more biomedcentral.com/submissions 